\title{
HUMAN AND RODENT TEMPORAL LOBE EPILEPSY IS CHARACTERIZED BY CHANGES IN O-GLCNAC HOMEOSTASIS THAT CAN BE REVERSED TO DAMPEN EPILEPTIFORM ACTIVITY
}

\author{
Richard G. Sanchez ${ }^{1}$, R. Ryley Parrish², Megan Rich ${ }^{1}$, William M. Webb ${ }^{1}$, Roxanne M. \\ Lockhart $^{1}$, Kazuhito Nakao ${ }^{1}$, Lara lanov ${ }^{3}$, Susan C. Buckingham ${ }^{1}$, Devin R. Broadwater ${ }^{4}$, \\ Alistair Jenkins, MD $^{5}$, Nihal C de Lanerolle ${ }^{6}$, Mark Cunningham ${ }^{2}$, Tore Eid $^{6}$, Kristen Riley ${ }^{7}$, \\ and Farah D. Lubin ${ }^{1, *}$ \\ ${ }^{1}$ Department of Neurobiology, University of Alabama - Birmingham, AL \\ ${ }^{2}$ Institute of Neuroscience, Newcastle University, Newcastle upon Tyne, UK \\ ${ }^{3}$ Civitan International Research Center, University of Alabama at Birmingham, Birmingham, AL \\ ${ }^{4}$ School of Medicine, University of Alabama - Birmingham, AL \\ ${ }^{5}$ Department of Neurosurgery, Royal Victoria Infirmary, Newcastle upon Tyne, NE1 4LP, UK \\ ${ }^{6}$ Department of Laboratory Medicine and of Neurosurgery, Yale School of Medicine - New Haven, \\ CT \\ ${ }^{7}$ Department of Neurosurgery, University of Alabama - Birmingham, AL
}

\section{Abstract}

Temporal Lobe Epilepsy (TLE) is frequently associated with changes in protein composition and post-translational modifications (PTM) that exacerbate the disorder. O-linked- $\beta$ - $N$-acetyl glucosamine (O-GlcNAc) is a PTM occurring at serine/threonine residues that is derived from and closely associated with metabolic substrates. The enzymes O-GlcNActransferase (OGT) and OGlcNAcase (OGA) mediate the addition and removal, respectively, of the O-GlcNAc modification. The goal of this study was to characterize OGT/OGA and protein O-GlcNAcylation in the epileptic hippocampus and to determine and whether direct manipulation of these proteins and PTM's alter epileptiform activity. We observed reduced global and protein specific OGlcNAcylation and OGT expression in the kainate rat model of TLE and in human TLE hippocampal tissue. Inhibiting OGA with Thiamet-G elevated protein O-GlcNAcylation, and decreased both seizure duration and epileptic spike events, suggesting that OGA may be a therapeutic target for seizure control. These findings suggest that loss of O-GlcNAc homeostasis in the kainate model and in human TLE can be reversed via targeting of O-GlcNAc related pathways.

*Corresponding author at: Department of Neurobiology, University of Alabama - Birmingham, 1825 University Boulevard, Birmingham, AL, 35294, United States.

Publisher's Disclaimer: This is a PDF file of an unedited manuscript that has been accepted for publication. As a service to our customers we are providing this early version of the manuscript. The manuscript will undergo copyediting, typesetting, and review of the resulting proof before it is published in its final citable form. Please note that during the production process errors may be discovered which could affect the content, and all legal disclaimers that apply to the journal pertain. 


\section{Keywords}

Hippocampus; Magnetic resonance imaging; Thiamet-G; Electroencephalogram;

Electrophysiology; Mass Spectrometry; post-translational modification; O-GlcNAcylation

\section{Introduction}

Temporal lobe epilepsy (TLE) is a neurological disorder characterized by recurrent, unprovoked seizures. Previous studies in TLE have revealed changes in cytoskeleton modifications, synaptic proteins, mitochondrial proteins, ion channels, and chaperone proteins $[1,2]$. Although proteomic studies have investigated the role of post-translational modifications (PTM) in these proteins, these studies have focused mainly on phosphorylation. Protein expression and function is a dynamic process that requires precise regulation in order to maintain cellular homeostasis under changing conditions. A cellular mechanism by which cells regulate these parameters is through PTM's, where enzymes add functional groups to modulate protein dynamics. In TLE, several of these modifications are disrupted, with the majority of studies to date revealing irregularities in the extent of protein phosphorylation and its downstream effects on neuronal homeostasis [3-6]. Similar to phosphorylation, O-GlcNAcylation, has recently gained attention for its role in neuronal excitability in acute brain slices from non-epileptic animals [7, 8]. Whether O-

GlcNAcylation plays a role in the regulation of chronic epileptic seizures and is involvement in protein regulation remains unknown.

O-GlcNAcylation depends on cellular metabolism in order to synthesize the substrate, UDPGlcNAc, which is then used by O-GlcNAc Transferase (OGT) to add O-GlcNAc to serine and threonine residues [9-11]. The removal of this modification is regulated by OGlcNAcase (OGA) [9-11]. Together, OGT and OGA regulate global levels of O-

GlcNAcylation across a variety of cellular stresses in order to preserve homeostasis [12]. Unlike phosphorylation, in which numerous kinases and phosphatases target many of the same proteins, O-GlcNAcylation relies on only OGT and OGA [13]. Importantly, OGA can be potently and selectively inhibited by Thiamet-G, a purine analog that can cross the bloodbrain barrier [14]. By inhibiting OGA, Thiamet-G increases global O-GlcNAcylation levels within eukaryotic cells [14]. Recently, several studies have focused on O-GlcNAcylation in neurological disorders such as Alzheimer's, Parkinson's, Huntington's, and other nervous system processes such as appetite, long-term depression (LTD), neuronal hyper-excitability and protein structure [15-24]. Prior work has demonstrated that altering O-GlcNAcylation can have a therapeutic effect on acutely induced seizure activity from non-epileptic animals but it remains unknown if alterations in O-GlcNAcylation are associated with epilepsy [7, 8]. Our current study focuses on this dynamic modification in chronically seizing epileptic animals that produce and sustain their own spontaneous seizures and are modeled more closely to human TLE. In addition to being able to observe these modifications in epileptic rodent models we were able to study these affects on ex vivo resected human TLE samples.

In the present study, we investigated the role of O-GlcNAcylation using a rodent model of TLE as well as human epileptic brain tissue, asking whether targeted manipulation of this 
modification could ameliorate epileptiform activity. We identified global decreases in OGlcNAcylation in epileptic rats and in human patients with TLE. Mass spectrometry analysis revealed that O-GlcNAcylation marks were dysregulated and expressed on specific proteins in the rat epileptic hippocampus compared to age-matched non-epileptic controls. Additional experiments revealed that inhibition of OGA using Thiamet-G resulted in reduced seizure duration and decreased interictal spike frequency. Similarly, ex vivo electrophysiological recordings from human TLE samples showed decreased interictal spike activity with OGA inhibition compared to recordings taken in vehicle-treated controls. Collectively, these results support a critical role for protein OGlcNAcylation in epilepsy and its novel therapeutic potential in the treatment of chronic seizures.

\section{Material and Methods:}

Antibodies:

The following antibodies were used: 1:500 anti-O-GlcNAc (CTD110.6,-MMS-248R from Covance, Princeton, NJ, USA), 1:500 anti-O-GlcNAc Transferase (O6264, Sigma, St. Louis, MO, USA), 1:20000 goat-anti-mouse (926-32350, Licor, Lincoln, NE, USA), 1:20000 goatanti-rabbit (926-32211, Licor), 1:1000 anti-Actin (ab1801, Abcam, Cambridge, UK), 1:1000 anti-NeuN (MAB377, Abcam), 1:1000 anti-GFAP (ab7260, Abcam).

\section{Kainate Treatment:}

Animals were injected with kainic acid (KA) [10 mg/kg; (Tocris Cookson Inc., Ellisville, MO, USA)] or saline (vehicle) intraperitoneally (IP). The severity of behavioral seizures following KA injection was scored according to the Racine scale [25]: a five-point scale which takes the five following behaviors as indicative of respectively increasing seizure severity: mouth and face clonus and head nodding (1); clonic jerks of one forelimb (2); bilateral forelimb clonus (3); forelimb clonus and rearing (4); forelimb clonus with rearing and falling (5). The onset of status epilepticus (SE) was defined as the time from KA injection to the occurrence of continuous seizure activity (Racine score 4-5) over a period of 4 hours. All control animals were handled in the same manner as the KA-treated animals but injected with saline. For tissue collection, the hippocampus was removed and oxygenated (95\%/5\% O2/CO2) in ice-cold cutting solution (110 mM sucrose, $60 \mathrm{mM} \mathrm{NaCl}, 3 \mathrm{mM} \mathrm{KCl}$, $1.25 \mathrm{mM}$ NaH2PO4, $28 \mathrm{mM} \mathrm{NaHCO} 3,0.5 \mathrm{mM} \mathrm{CaCl} 2,7 \mathrm{mM} \mathrm{MgCl} 2,5 \mathrm{mM}$ glucose, 0.6 $\mathrm{mM}$ ascorbate). The cornu ammonis (all CA regions), and the dentate gyrus (DG) region were microdissected and frozen immediately on dry ice. The hippocampus was bisected with the dorsomedial half being divided into four pieces. Using anatomic landmarks, each piece was dissected into CA and DG region. The CA and DG were dissected with a cut along the hippocampal fissure. The tissue was then stored at $-80^{\circ} \mathrm{C}$ for RNA and DNA extraction.

\section{Electroencephalogram (EEG):}

4 weeks following the administration of kainic acid, rats underwent an electrode implantation. Electrodes (MS333/1-B/SPC, Plastics One, Raonoke, VA, USA) for EEG recordings were trimmed to $1.75 \mathrm{~mm}$ in length and fitted into three holes so that they contacted the dura and the connector was flush with the skull. The ground wire was placed into the most caudal hole. For EEG recordings, animals were transferred to individual 
housing in custom-designed and constructed Plexiglas cages at 5 weeks. EEG data were acquired using 8 Biopac Systems amplifiers and AcqKnowlege 4.1 EEG Acquisition and Reader Software (BIOPAC Systems, Inc., Goleta, CA, USA). Data were stored and analyzed in digital format. Each cage was also equipped with an IR Digital Color CCD camera (Lorex Technology, Inc., Linthicum, MD, USA) and animals are recorded concurrently with EEG monitoring. Baseline recordings were assessed for $24 \mathrm{hrs}$ then $10 \mathrm{mg} / \mathrm{kg}$ of Thiamet-G dissolved in $0.1 \%$ w/v saline (SD Chemmolecules, Owings Mills, MD, USA) was administered intraperitoneal (I.P) and then $10 \mathrm{mg} / \mathrm{kg}$ after each post-injection. Both saline and kainic acid, cohorts were injected with Thiamet-G. After 24 hrs of EEG recording postinjection, a second treatment with the same dosage was administered. Animals were recorded via EEG for $24 \mathrm{hrs}$ after each injection of Thiamet-G. Animals received a total of three independent treatments at same dosage of the drug.

Tissue for Western blots was collected from 4 weeks of age using the previously-described methods. The whole hippocampus was collected and then sub-dissected. All EEG data were analyzed manually using Matlab by an observer blinded to the sample's identity.

Abnormalities in the recordings indicative of epileptic activity are aligned chronologically with the corresponding video in order to confirm seizures.

\section{Immunofluorescence:}

Animals were sacrificed by rapid decapitation; brains were removed, and fixed in $4 \%$ paraformaldehyde overnight at $4^{\circ} \mathrm{C}$. The next day the samples were washed with $1 \mathrm{x}$ PBS 5x five minutes each time before incubating with $30 \%$ sucrose $(\mathrm{w} / \mathrm{v})$ overnight at $4^{\circ} \mathrm{C}$. The tissue was then flash frozen on dry ice and mounted in O.C.T. (VWR, Randor, PA, USA) 10micron Sections $(10 \mu \mathrm{M})$ were taken throughout the dorsal hippocampus and mounted onto slides. Antigen retrieval was achieved by boiling in citric acid buffer followed by washing in 1x PBS. Slices were then blocked for $1 \mathrm{hr}$ (4\% normal goat serum, 4\% normal donkey serum and $0.3 \%$ Triton-X in PBS) and incubated in primary antibody for O-GlcNAc (1:200 CTD110.6, MMS-248R, Covance), and NeuN (1:1000, MAB377, Millipore), overnight at $4{ }^{\circ} \mathrm{C}$. The following day sections were rinsed with 1x PBS and incubated in Alexa Fluor 488labeled (1:500, \#111-545-003, Jackson Immuno Research, West Grove, PA, USA) or Rhodamine-labeled (TRITC, 1:500, \#715-025-150, Jackson Immuno Research) secondary antibodies for $2 \mathrm{hrs}$ and, rinsed with 1x PBS and then cover slipped with Vectashield mounting media with DAPI (H-1500, Vector Laboratories, Burlingame, CA, USA). Images were taken on a Zeiss Axio Imager microscope and analyzed using Image $\mathrm{J}$.

\section{Human Tissue Samples:}

Pharmacologically-resistant hippocampal and cortical tissue samples from human TLE patients were provided by Tore Eid, MD from the Departments of Laboratory Medicine and of Neurosurgery, at Yale School of Medicine. Additional tissue was provided by Kristen O. Riley, MD from the Department of Neurosurgery and Yancy G. Gillespie, MD from the Wallace Tumor Institute at UAB. Acquisition and processing of control human tissue were performed by the Alabama Brain Collection (ABC) https://www.uab.edu/medicine/ psychiatry/research/resources-0/alabama-brain-collection. There was no correlation between postmortem interval (PMI) and protein levels from the ABC samples based on a Pearson's 
correlation [26, 27]. Patient demographics and pharmacological history are described in Supplementary Table 2.

\section{Small Animal Magnetic Resonance Imaging:}

$T_{1^{-}}$and $T_{2}$-weighted images were collected on a 9.4T Bruker BioSpin horizontal small bore animal MRI scanner. The imaging parameters were set as follows: $1 \mathrm{~mm}$ slice thickness, 1 $\mathrm{mm}$ between slice distance, $0.1 \times 0.1 \times 1 \mathrm{~mm}$ voxel size, $30 \times 30 \mathrm{~mm}$ FOV, 27 images per acquisition. T2-weighted hippocampal intensities were normalized to within-slice cortical intensity using ImageJ software ( $\mathrm{n}=5 /$ group).

\section{Western Blotting:}

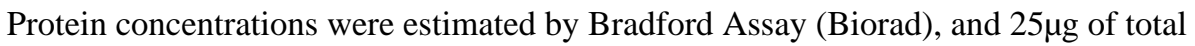
protein/sample was reduced in 5x sample loading buffer (0.1 M Tris-HCl, 4\% SDS, $20 \%$ glycerol, $0.2 \% \beta$-mercaptoethanol, $0.2 \%$ bromphenol blue), boiled for $10 \mathrm{~min}$, separated by $10 \%$ SDS-PAGE, and transferred onto PVDF membranes using Trans-Blot Turbo transfer system (1704155, BioRad, Hercules, CA, USA). Membranes were activated with methanol for three minutes before transfer, blocked for $1 \mathrm{hr}$ at room temperature and incubated overnight at $4^{\circ} \mathrm{C}$ with primary antibodies following the transfer. Three washes were performed with 1x PBST (PBS and $0.01 \%$ Tween) between primary and secondary antibodies and after stripping. The membranes were blocked with 1:1 Licor Blocking buffer (P/N 927-40003, Licor) and PBST for one hour at room temperature after transfers and stripping. Imaging was performed using Licor Odyssey scanner at 700/800 channel, and Licor Odyssey software. Image analysis was performed using Image Studio Lite Ver. 3.1.

\section{Sample Preparation for mass spectrometry:}

Protein was extracted from rat dorsal hippocampus CA using M-PER (78501, Thermo Fisher Scientific) and quantified using Pierce BCA Protein Assay Kit (23225, Thermo Fisher Scientific). Extracts were diluted in LDS PAGE buffer (NP0007, Invitrogen) followed by reduction, heat denaturing, and separation on an SDS Bis-Tris gel (4-12\%, NP0323BOX, Invitrogen). The gels were stained overnight with colloidal blue (89871, Invitrogen). The entire lane comprising each sample was cut into $12 \mathrm{MW}$ fractions and equilibrated in 100 $\mathrm{mM}$ ammonium bicarbonate ( $\mathrm{AmBc})$. Gel slices were reduced, carboxymethylated, dehydrated, and digested with Trypsin Gold (V5280, Promega, Madison, WI, USA) as per manufacturers' instructions. Following digestion, peptides were extracted, the volume was then be reduced in a SpeedVac to near dryness, and resuspended to $20 \mu \mathrm{l}$ using $95 \% \mathrm{ddH}_{2} \mathrm{O} /$ $5 \% \mathrm{ACN} / 0.1 \%$ formic acid (FA) prior to analysis by 1D reverse phase LC-ESI-MS2 (as outlined below).

\section{HPLC-electrospray tandem mass spectrometry:}

Peptide digests were injected onto a 1260 Infinity HPLC stack (Agilent, Santa Clara, CA, USA) and separated using a 75 micron I.D. x $15 \mathrm{~cm}$ pulled tip C-18 column (00G-4053-E0, Jupiter C-18 $300 \AA$ A, 5 micron, Phenomenex, Torrance, CA, USA). This system runs in-line with a Thermo Orbitrap Velos Pro hybrid mass spectrometer, equipped with a nanoelectrospray source (Thermo Fisher Scientific), and all data was collected in CID mode. The 
HPLC was configured with binary mobile phases that include solvent A $(0.1 \% \mathrm{FA}$ in $\left.\mathrm{ddH}_{2} \mathrm{O}\right)$, and solvent $\mathrm{B}\left(0.1 \% \mathrm{FA}\right.$ in $\left.15 \% \mathrm{ddH}_{2} \mathrm{O} / 85 \% \mathrm{ACN}\right)$, programmed as follows;

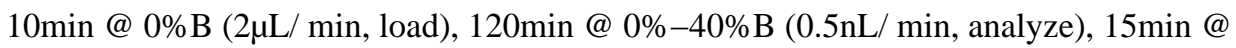
$0 \% \mathrm{~B}(2 \mu \mathrm{L} / \mathrm{min}$, equilibrate). Following each parent ion scan $(350-1200 \mathrm{~m} / \mathrm{z} @ 60 \mathrm{k}$ resolution), fragmentation data (MS2) was collected on the topmost intense 15 ions. For data dependent scans, charge state screening and dynamic exclusion were enabled with a repeat count of 2 , repeat duration of $15.0 \mathrm{~s}$, and exclusion duration of $60.0 \mathrm{~s}$.

\section{Mass Spectrometry Data Conversion and Searches:}

The XCalibur RAW files were collected in profile mode, centroided and converted to MXML using ReAdW v. 3.5.1. The mgf files were then created using MzXML2Search (included in TPP v. 3.5) for all scans. The data was searched using SEQUEST, which was set for two maximum missed cleavages, a precursor mass window of 20ppm, trypsin digestion, variable modification $C$ at 57.0293, and M at 15.9949. Searches were performed with a species-specific subset of the UniRef100 database.

\section{Peptide Filtering, Grouping, and Quantification:}

The list of peptide IDs generated based on SEQUEST search results were filtered using Scaffold (Protein Sciences, Portland, OR, USA). Scaffold filters and groups all peptides to generate and retain only high confidence IDs while also generating normalized spectral counts (N-SC's) across all samples for the purpose of relative quantification. The filter cutoff values were set with minimum peptide length of $>5 \mathrm{AA}$ 's, with no $\mathrm{MH}+1$ charge states, with peptide probabilities of $>80 \%$ C.I., and with the number of peptides per protein $\geq 2$. The protein probabilities are then set to a $>99.0 \%$ C.I., and an FDR $<1.0$. Scaffold incorporates the two most common methods for statistical validation of large proteome datasets, the false discovery rate (FDR) and protein probability [28-30]. Relative quantification across experiments were then performed via spectral counting, and when relevant, spectral count abundances were then normalized between samples [31-33].

\section{Proteomics Analysis:}

For the proteomic data generated, two separate non-parametric statistical analyses were performed for each pair-wise comparison. These non-parametric analyses include 1) the calculation of weight values by significance analysis of microarray (SAM; cut off $>|0.6|$ combined with 2) T-Test (single tail, unequal variance, cut off $\mathrm{p}<0.05$ ), which then were sorted according to the highest statistical relevance in each comparison. For SAM, whereby the weight value $(\mathrm{W})$ is a statistically derived function that approaches significance as the distance between the means $(\mu 1-\mu 2)$ for each group increases, and the SD $(\delta 1-\delta 2)$ decreases using the formula, $W=(\mu 1-\mu 2) /(\delta 1-\delta 2)[34,35]$. For protein abundance ratios determined with N-SC's, we set a 1.5-2.0 fold change as the threshold for significance, determined empirically by analyzing the inner-quartile data from the control experiment indicated above using $\ln -\ln$ plots, where Pierson's correlation coefficient (R) was 0.98 , and $>99 \%$ of the normalized intensities fell between $+/-1.5$ fold. In each case, any two of the three tests (SAM, Ttest, or fold change) had to pass. 
Gene ontology assignments and pathway analysis were carried out using MetaCore (GeneGO Inc., St. Joseph, MI, USA). In addition, the final proteins list is analyzed using the auto-expand algorithm within MetaCore using the default setting (i.e. expanded by 50 nodes). In parallel, the expand-by-one algorithm is used to identify connections to the neighboring proteins, known drug interactions, and any known correlation to a disease, or specific biological process. Interactions identified within MetaCore are manually correlated using full-text articles. Detailed algorithms have been described previously [36, 37]. The heatmap was constructed in R with the package 'ComplexHeatmap' (v 1.18.0) and the scatter plots were created with 'ggplot2' (v 2.2.1.9000).

\section{Human Electrophysiology:}

The electrophysiological data obtained from ex vivo slice studies were derived from patients with medically intractable epilepsy undergoing elective neurosurgical tissue resection for the removal of a sclerotic hippocampus. All patients gave their informed consent, before surgery, for the use of the resected brain tissue for scientific studies. This study was approved by the Newcastle and the North Tyneside 2 Local Research Ethics Committee (06/ Q1003/51) (date of review 03/07/06) and had clinical governance approved by the Newcastle Upon Tyne Hospitals NHS Trust (CM/PB/3707). Slices were prepared from these samples using methods as previously described [38-40]. The time between resection and slice preparation was $<5$ min. Extracellular recordings (DC-500 Hz) were conducted with ACSFfilled glass microelectrodes ( $2 \mathrm{M} \Omega$ ) connected to an extracellular amplifier (EXT-10-2F, npi electronic $\mathrm{GmbH}$, Tamm, Germany). Signals were digitized $(5 \mathrm{kHz})$ and recorded on a computer and then extracellular field recordings were analyzed to detect events using a custom-written code in Matlab2015b (Mathworks, MA, USA).

siRNA Infusion:

For electrophysiological studies, hippocampal slices were collected from 6-8 week old, male Sprague-Dawley rats as previously described [6]. All rats had previously undergone stereotactic cranial infusion of siRNA according to previously described methods [41]. Briefly, animals were anesthetized by way of intraperitoneal injection of dexmedetomidineketamine and received bilateral infusions of Accell SMARTpool siRNAs (Thermo) targeting either OGT (\#E-080125-00-05) or scrambled, negative controls (\#D-001910-10-05) in the dorsal hippocampus using the following stereotaxic coordinates relative to bregma: $\mathrm{A} / \mathrm{P}$ $-3.6 \mathrm{~mm}, \mathrm{M} / \mathrm{L} \pm 1.7 \mathrm{~mm}, \mathrm{D} / \mathrm{V}-3.6 \mathrm{~mm}$. Infusions were delivered at a constant rate of $0.1 \mathrm{uL}$ per minute using a linear actuator for a total volume of $1 \mathrm{uL}$ per side. Non-targeting, fluorescent Accell siRNA (\#D-001960-01) were used to confirm targeted delivery of siRNA to the dorsal hippocampus. For all conditions, fresh stocks of siRNA (100 $\mu \mathrm{M})$ were resuspended in Accell siRNA resuspension buffer to a concentration of $4.5 \mu \mathrm{M}$ immediately prior to surgery.

\section{Electrophysiology:}

Following surgery, each rat was allowed five days of recovery time after which its brain was harvested and hippocampal slices were collected for further testing. High-frequency stimulation of the Schaffer collateral/commissural pathway (CA3-CA1) was conducted using four trains of 100 pulses at $100 \mathrm{~Hz}$, spaced 60 seconds apart. The initial slope of the 
field excitatory postsynaptic potential (EPSP) was measured as an index of synaptic strength. Percent fEPSP slopes were averaged after $20 \mathrm{~min}$ of baseline recording. Electrophysiological data are reported as means \pm SEM, where $\mathrm{n}$ represents the number of slices.

\section{Statistical Analysis for Biochemistry studies:}

Data is expressed as mean \pm S.E.M and compared by a Student-test and Mann-Whitney. Shapiro-Wilk and Kolmogorov-Smirnov statistics were performed to take into account any age, sex, race, and post-mortem interval information, none of the listed factors are contributing to our results for the OGT or O-GlcNAc protein levels for the human experiments. Statistically significant differences between groups were defined as $\mathrm{p}<0.05$.

\section{Results}

\section{Hippocampal O-GIcNAcylation and OGT protein levels decreased in epileptic rats}

KA-induced epilepsy has been shown to alter a variety of PTMs in proteins of the hippocampus. Therefore we sought to quantify global O-GlcNAcylation levels in the hippocampus eight weeks post-SE when the animals had become fully epileptic and experienced self-convulsive seizures (Fig. 1a). Analysis of protein O-GlcNAcylation in the CA regions of the hippocampus revealed significant decreases of O-GlcNAcylation in epileptic animals compared to controls $\left(\mathrm{t}_{(4)}=13.02, p=0.0002, \mathrm{t}_{(8)}=2.363, p=0.0457\right.$; Fig. 1bc). To investigate this decrease further, we measured OGT protein levels in the same region and observed a significant decreased in OGT protein levels in the epileptic rats when compared to controls $\left(\mathrm{t}_{(4)}=13.02, p=0.0002, \mathrm{t}_{(8)}=2.363, p=0.0457\right.$; Fig. 1d). In light of these results, we wanted to understand whether loss of OGT contributed to neuronal hyperexcitability. Following siRNA-mediated knockdown of OGT and high-frequency stimulation of the Schaffer collateral/commissural pathway in wild-type rats (Supplemental Fig. 1a-c), we detected a trend toward increasing percent fEPSP and fEPSP slope. Our findings suggest an increased rate of neuronal firing with reduction of OGT. Meanwhile, no changes were detected in the paired-pulse facilitation between groups, indicating that any changes in neuronal firing were due to changes in the postsynaptic neuron. Taken together, these results indicate a reduction of O-GlcNAc and OGT protein levels in the epileptic hippocampus and suggest a correlation between epilepsy and protein O-GlcNAcylation.

Next, we sought to investigate specific proteins that displayed differential O-GlcNAcylation expression associated with TLE pathology. Using HPLC-electrospray tandem mass spectrometry we measured the abundance of proteins that had differentially altered OGlcNAcylation expression in the CA regions of the hippocampus at eight weeks post-SE. We found that 59 proteins were differentially expressed in TLE. Within those 59 proteins, only 17 proteins had O-GlcNAc modifications with 9 of which that were differentially OGlcNAcylated. Gene ontology analysis revealed that the majority of diseases associated with differential expression of these proteins were neurodegenerative or cytoskeletal in nature. Additionally, among these 17 proteins, 12 had been associated with epilepsy in previous studies [8] (Supplementary Table1) (Fig. 2A). 
In addition to measuring O-GlcNAcylation, we also measured phosphorylation and discovered increases in protein phosphorylation, particularly on those proteins that had shown decreases in protein O-GlcNAcylation. We next analyzed overall protein expression against protein phosphorylation and protein O-GlcNAcylation (Supplemental Fig. 2a-b) revealing two distinct cluster groups. These clusters indicated that increased protein expression was positively correlated with increased protein phosphorylation, and only a few of the more highly expressed proteins also had changes in O-GlcNAcylation. In contrast to phosphorylation, increased protein O-GlcNAcylation was predominantly present on proteins that showed decreased expression. These clusters persisted when O-GlcNAcylation and phosphorylation were plotted against each other (Supplemental Fig. 2c). The Z-scores were plotted from each biological replicate against either modification to demonstrate the contrast between their fold change (Supplemental Fig. 2d-e). Taken together our mass spectrometry analysis corroborated our findings that overall protein O-GlcNAcylation was decreased in the epileptic animal hippocampus while highlighting the particular proteomic ontologies affected by this loss. Additionally, our findings revealed that certain proteins actually show increased O-GlcNAcylation in the epileptic hippocampus. Collectively, these findings provide evidence that differentially expressed proteins and changes in PTMs are associated with TLE and other disease states highlighting the importance of protein PTM in homeostasis.

\section{OGA inhibition via acute Thiamet-G treatment reduced epileptiform activity in the epileptic hippocampus.}

The observed global loss of O-GlcNAcylation and OGT prompted additional experiments to determine the role of this PTM in epilepsy. Using the KA model of epilepsy, we recorded brain neuronal activity with EEG one month post-SE. We then administered Thiamet-G $(10 \mathrm{mg} / \mathrm{kg} /$ day), a known OGA inhibitor used to increase O-GlcNAcylation, once a day for three consecutive days in order to measure its effect on epileptiform activity (Fig. 3a). We measured baseline EEG activity between control animals and epileptic animals and found that epileptic animals demonstrated higher power than the controls indicating more epileptiform activity or the integral average of the amplitude of the EEG signal across time (Fig. 3b-c). The epileptic rats presented with more sharp spikes and larger amplitudes of voltage than the control animals, suggesting synchronous activity or seizures in the spectrogram depicted with warmer colors. The epileptic animals then underwent a daily regimen of OGA inhibition for three days while being monitored with EEG (Fig. 3d). Following three days of Thiamet-G treatment, epileptic rats displayed a reduction in epileptiform activity displaying decreases in sharp spikes and voltage amplitudes as well as a decrease in the seizure frequency and duration $\left(\mathrm{t}_{(7)}=1.999, p=0.858, \mathrm{t}_{(34)}=3.497, p=0.0013\right.$; Fig. 3e-f). We then assessed different wave oscillations from EEG recordings in order to determine if there were any changes in band power between frequencies for each day of Thiamet-G treatment. (Fig. 3g). The frequencies were divided into the lower band frequencies, such as delta and theta waves, which are associated with sleep, and the higher frequency bands such as gamma, which is more closely associated with consciousness and attentiveness [42]. These bands can be used to characterize seizure severity [43-45]. In this study, OGA inhibition helped restore the power of the lower frequencies (delta-alpha) more so than the higher frequencies (beta-gamma) to the baseline of the control group. 
We furthered analyzed each frequency type against their relative power. As expected the largest powers for each given frequency band were from the epileptic rat recordings prior to Thiamet-G treatment $(\mathrm{t}(32-52)=, \mathrm{p}=0.0016-<0.0001 ;$ Fig. $3 \mathrm{~h})$. By the first day of treatment, these bands showed a reduction in power and began to mirror the power levels of the nonepileptic rats, with the exception of the gamma frequency. The gamma frequency was the least affected by treatment; this could be explained by a local measure of activity and not by an overall global cortical network due to a single measurement of activity with an electrode. With each day of treatment, the relative power of each band decreased with the exception of the theta band which plateaued after the first treatment of Thiamet-G. The excitatory regular spiking, and pyramidal neuron intrinsic bursting typically characterize this band, suggesting that inhibition of OGA via Thiamet-G may preferentially target this group of neurons.

\section{Chronic inhibition of OGA activity increased hippocampal atrophy in epileptic rats}

Since OGA inhibition dampened epileptiform activity and seizure duration in a wide spectrum of frequencies, we sought to determine if there were any morphological changes associated with Thiamet-G treatment over a prolonged period of usage. Hippocampal scarring and/or gliosis is often observed in animal models of TLE as well as in humans, where it leads to hippocampal atrophy [46-49]. Hippocampal atrophy in TLE patients has been observed using MRI $T_{2}$ weighted scans where the ventricles adjacent to the hippocampus expand along with a reduction of hippocampal size [50-52]. We created epileptic rats as previously described, and performed $\mathrm{T}_{2}$ weighted MRI eight weeks post KA in order to record their ventricular volumes prior to Thiamet-G treatment. We then began a two-week treatment regimen for these animals as well as non-epiletic controls with either saline or Thiamet-G $(10 \mathrm{mg} / \mathrm{kg} / \mathrm{day})$ and measured their ventricular volumes after treatment (Fig. 4a-c One way ANOVA, $\mathrm{F}=10.05, p=0.0002$ ). Epileptic rats displayed significantly higher voxel area units prior to treatment compared to non-epileptic controls. Following two weeks of treatment, voxel area increased in both Thiamet-G treated rats and saline-treated epileptic rats indicating no restoration of hippocampal morphology with Thiamet-G treatment. With the most significant ventricle increases observed in Thiamet-G treated epileptic rats. These scans suggest that Thiamet-G does little to inhibit or slow the progression of ventricular expansion seen in epilepsy [52-55].

Following MRI scans, animals were sacrificed and brain tissue was processed for immunohistochemistry experiments. We stained brain slices for O-GlcNAcylation (Supplemental Fig. 3b) as an output for Thiamet-G treatment. We observed increases in OGlcNAcylation with Thiamet-G treatment in the hippocampus particularly in the molecular layer in both control and epileptic rats that received Thiamet-G. Taken together, these experiments suggest that OGA inhibition does not stop or reverse epileptic hippocampal atrophy. These findings leave open the possibility that Thiamet-G treatment may slow the progression of hippocampal atrophy if it is begun earlier in the disorder. However, OGA inhibition at a chronic seizure state does not appear to restore atrophied tissue.

Because Thiamet-G treatment resulted in increased O-GlcNAcylation expression in epileptic animals, we next sought to understand how chronic treatment with Thiamet-G would affect O-GlcNAcylation levels on proteins shown to be O-GlcNAcylated and differentially 
expressed in TLE. Specifically, we wanted to ask whether OGA's expression was altered in epilepsy, and if so, whether Thiamet-G treatment restored OGA expression to homeostatic levels. We first looked at OGA protein expression in our epileptic animals that were treated for two weeks with Thiamet-G, (One way ANOVA F=1.852 p=0.085, Fig. 3d-e). We noticed no significant changes in OGA protein expression in control animals treated with ThiametG. Although not significant, we did notice a trend in increased OGA protein expression in epileptic animals. When these animals were treated with Thiamet-G, levels of OGA expression resembled those of saline-treated controls.

Based on our proteomic analysis (Fig. 2a), we identified Sortilin-Related Receptor (SORL1) and tropomodulin $2(\operatorname{Tmod} 2)$ as proteins that undergo increased and decreased protein OGlcNAcylation in TLE, respectively (Supplemental Fig. 4). SORL1 is a receptor that binds to LDL and transports it into the cells via endocytosis, a process that is subject to inhibition upon binding to the receptor-associated protein (RAP) [56]. SORL1 has also been implicated in APP trafficking to and from the Golgi apparatus in Alzheimer's disease [57, 58]. Tmod 2 is an actin-binding protein that stabilizes ADP-bound actin monomers onto actin filaments and is downregulated in epilepsy [59, 60]. To test the effect of Thiamet-G administration on these proteins' PTMs we used immunoprecipitation followed by Western blot to investigate the levels of O-GlcNAcylaion on these proteins specifically. We observed no differences in O-GlcNAcylation on immunoprecipitated SORL1, nor did we observe any difference in its association with OGT (Fig. 4f). Immunoprecipitation of Tmod2 revealed slight increases in O-GlcNAcylation in animals treated with Thiamet-G, along with decreases of total O-GlcNAcylation in the inputs, or the raw unimmunoprecipitated samples (Fig. 4g). Furthermore, no differences were observed in the degree of association between Tmod2 and OGT. These results suggest that Thiamet-G cannot restore the decreased levels of O-GlcNAcylation of SORL1 and Tmod2 specifically in epileptic rats.

\section{Deficits in O-GIcNAcylation and OGT in patients with TLE}

Our initial rodent studies have shown that O-GlcNAcylation and OGT are downregulated in the hippocampi of epileptic rats, but we were unsure of how O-GlcNAcylation and OGT might behave in human TLE tissue (Supplementary Table2). We began by measuring OGlcNAcylation and OGT expression in resected human hippocampus from TLE patients and compared them to age-matched controls from post-mortem human hippocampal tissue (Fig. 5a). We observed a significant loss of O-GlcNAcylation and OGT expression $\left(\mathrm{t}_{(18)}=3.198\right.$, $p=0.0050, \mathrm{t}_{(11)}=1.941, p=0.0783 \mathrm{Fig}$. 5bc) in TLE patient tissue compared to control postmortem tissue as seen in our epileptic rats. We next asked whether SORL1 and Tmod2 were being modified in the same manner that we had seen in our epileptic rats. We immunoprecipitated SORL1 (Fig. 5d) and Tmod2 (Fig. 5e) from human postmortem samples and similar to our results in rodent tissue, we observed no differences in OGlcNAcylation of the proteins, nor any differences in their interaction with OGT.

\section{OGA inhibition decreased spike events and increased OGT and OGA protein expression in human TLE resected tissue}

Because we observed decreases in O-GlcNAcylation levels in samples from human TLE patients, we next sought to test whether bath application of Thiamet-G could reduce 
epileptiform activity in live TLE patient tissue. As previously described [38], baseline spontaneous interictal-like activity was recorded for $1 \mathrm{hr}$, after which, slices were then exposed to Thiamet-G (100 $\mu \mathrm{M})$ (Fig. 6a). Prior to treatment with Thiamet-G, tissue slices exhibited spontaneous interictal-like activity at an average of 66 spikes per minute $(+/-6.8$ spikes/minute), a rate which decreased to an average of 40 spikes per minute $(+/-8.9$ spikes/ minute) after one hour of Thiamet-G treatment (Fig. 6b-d).

Interestingly, our human electrophysiological data revealed no immediate decreases in epileptiform activity following Thiamet-G administration indicating that it is most likely functioning through PTM of proteins altering their structure and function over time as oppose to direct interactions with ionic channels/receptors. Importantly, slices that were not treated with Thiamet-G had an average of 78 spikes per minute (+/- 4.8 spikes/minute), with no change in average spikes over time, demonstrating that time cannot account for the decrease in spikes per minute observed with Thimaet-G treatment. Thus, application of Thiamet-G to spontaneous hyper-excitable human epileptic tissue significantly decreased spike frequency.

We next examined the O-GlcNAcylation, OGT, and OGA levels in these tissues (Fig. 6e). Protein O-GlcNAcylation generally increased with Thiamet-G treatment in comparison to untreated resected tissue (Fig. 6f). OGT and OGA, which is enzymatically inhibited by Thiamet-G, increased with Thiamet-G treatment. Overall, we found that O-GlcNAcylation and OGT levels decreased in epilepsy, but promoting this PTM pharmacologically resulted in decreased seizure frequency and spiking, as well as increased protein O-GlcNAcylation.

\section{Discussion}

The role of protein O-GlcNAcylation in the epileptic hippocampus is yet to be determined. The current study demonstrates that O-GlcNAcylation and OGT is decreased in the hippocampus from a rodent model of epilepsy, and in resected human TLE tissue. Using the OGA inhibitor Thiamet-G, we were able to increase bulk protein O-GlcNAcylation levels, not only in the rodent epileptic hippocampus, but in resected human hippocampal TLE tissue as well. Pharmacologically increasing bulk protein O-GlcNAcylation levels led to reduced seizures and epileptiform activity in in the rodent epileptic hippocampus and in resected tissue from human TLE patients. Together, these results suggest that an imbalance in protein O-GlcNAcylation in the epileptic hippocampus can be reversed to lessen seizure episodes. Moreover, we demonstrate that the highly selective OGA inhibitor Thiamet-G can cross the blood-brain barrier to promote protein O-GlcNAcylation in the hippocampus, which provides a potentially novel and viable therapeutic option for epilepsy treatment.

Our finding of promoting protein O-GlcNAcylation decreases epileptiform activity is further supported by prior studies investigating O-GlcNAcylation in non-epileptic seizure models suggesting that this PTM may be involved in the maintenance of seizure activity [7, 8]. For example, with the epileptic KA model, O-GlcNAcylation levels, initially increased and subsequently decreased several hours post-SE [8]. Alternatively, pentylenetetrazol (PTZ) induction of non-epileptic seizures produced no changes in bulk O-GlcNAcylation levels, yet treatment with Thiamet-G decreased epileptiform activity following PTZ induced 
seizures [7]. Collectively, these prior studies demonstrate differential regulation of the OGlcNAc axis in non-epileptic seizures compared to epileptic seizures, indicating that epileptogenesis and etiology of seizures with respects to the OGlcNAc axis is worth noting. Thus, our current study expands on these prior findings by providing insight into the proteomic manner in which protein O-GlcNAcylation affects the epileptic hippocampus. Moreover, we provide evidence that O-GlcNAcylation protein may diminish seizures and epileptiform activity by targeting this modification to specific proteins altered in epilepsy.

Although its exact role is unclear, the O-GlcNAc axis has been shown to play a role in synaptic plasticity. A recent study demonstrated that pharmacologically elevating or reducing protein O-GlcNAcylation levels failed to alter basal hippocampal synaptic firing at the Schaffer collaterals in slices [61]. However, in vivo elevation of O-GlcNAc enhanced long term potentiation (LTP) and inhibition of O-GlcNAc resulting in a reciprocal affect [61]. This phenomena was attributed to increases in phosphorylation of SynapsinI/II due to O-GlcNAc elevation. Another study observed similar results with LTP and reveal that inhibition of OGT promoted LTP through translocation of AMPA receptor subunits (GluR1, and GluR2) to the plasma membrane [62]. Our siRNA knockdown of OGT studies (supplementary Figure 1) recapitulate these prior observations, suggesting that loss of OGT in epilepsy may be facilitating basal synaptic transmission at the synaptic level. Moreover, a recent study indicate that Thiamet-G treatment resulted in AMPA receptor GluA2 OGlcNAcylation and promoted NMDA receptor and protein kinase C-independent long term depression (LTD) in hippocampal slices [21]. Together, these findings present a potential mechanism by which Thiamet-G decreases epileptiform activity observed in our human resected TLE hippocampus.

Our human electrophysiological data reveal decreases in epileptiform activity following Thiamet-G administration indicating that it may be functioning through PTM of proteins altering their structure and function over indirect interactions with ionic channels/receptors. Interestingly, we observed that acute treatment with Thiamet-G did not result in an immediate decrease in seizure activity in our epileptic rodents that underwent EEG recordings (Figure 3). Instead, our EEG recordings revealed that it was only after three days of Thiamet-G treatment that the drug resulted in decreases in epileptiform brain activity. Collectively, these data suggest that bath application of Thiamet-G onto brain slices compared to in vivo administration has a timed-delay. Thus, Thiamet-G had to first cross the blood brain barrier to inhibit OGA, elevate global protein O-GlcNAcylation, and alter the proteomic landscape that resulted in decreased epileptiform activity.

It has not evaded our attention that although we observed decreased bulk protein OGlcNAcylation levels in the epileptic hippocampus, this is not necessarily reflective of decreased OGlcNAc at all individual proteins. In particular, proteomics analysis revealed that SORL-1, a multifunctional endocytic receptor, had increases in O-GlcNAcylation in the epileptic hippocampus. Immunoprecipitations studies revealed that OGA inhibition, which promotes increases in global OGlcNAcylation, resulted in loss of this PTM at the SORL-1 protein. Much like phosphorylation, O-GlcNAcylation can have structural and functional consequences on proteins that can enhance its overall function/activity or mute it [63, 64]. The role of 1980S-OG on SORL-1 is unknown at the moment but given that it was identified 
in untreated epileptic tissue, it may be perturbing its function in epilepsy to contribute to the hyper excitability observed in this disorder. Furthermore, SORL-1 has a plethora of sites that have been identified to be phosphorylated, and it has been established that there is extensive crosstalk between phosphorylation and O-GlcNAcylation [65-67]. One particular study from Wang et al. showed that phosphorylation sites from hundreds of peptides where not actively recycled, but with increases in O-GlcNAcylation it caused major losses of phosphorylation, however increases were observed as well [66]. Resonating the interplay and competition between phosphoproteome and O-GlcNAc proteome specific kinases such as GSK3 $\beta$ have been demonstrated to increase O-GlcNAcylation of many cytoskeletal and heat shock proteins when GSK3 $\beta$ was inhibited, and at the same time demonstrating decreases in nuclear protein O-GlcNAcylation [67]. Notably, the bi-directionality of this PTM and interplay with phosphorylation can serve as a biomarker of epilepsy and potentially as a measure of treatment options that result in decreases in epileptiform activity.

O-GlcNAcylation is critical in modulating cellular homeostasis; aberrant O-GlcNAcylation can result in inappropriate protein trafficking, degradation, transcription, translation, and overall function that could lead to cellular death [23, 68, 69]. Our study suggest that loss of O-GlcNAc homeostasis in epilepsy may affect the neuronal cytoskeleton with loss of OGlcNAc on Tmod2, as well as protein endocytosis/signaling with SORL1 demonstrating an alternative PTM profile in disease and in treatment. Furthermore, neuronal death is suggested in our $\mathrm{T}_{2}$ weighted MRI images, where epileptic animals that were administered chronic Thiamet-G treatment in displayed increases in ventricle size and hippocampal atrophy. Tmod2 has been previously described to be altered in Down syndrome, TLE, postseizures, and post-stroke, but its PTMs in these disorders have not yet been characterized $[59,60,70,71]$. Our study proposes that Tmod 2 may be functionally compromised in epilepsy due to its loss of O-GlcNAc at specific residues, and this may also be the case in the other neurological disorders where Tmod2 dysfunction has been implicated [59, 60, 70, 71].

Present TLE medications predominantly target ion channels that are expressed ubiquitously throughout the body, are associated with multiple side effects, and have a 66-75\% success rate in controlling seizures with patients. In light of current and previous findings, OGlcNAcylation provides a promising new therapeutic target in epilepsy or TLE and other chronic seizures disorders [9-11]. O-GlcNAcylation's immersive role in cellular metabolism, allows it to be regulated by the availability of glucose, amino acids, fatty acids, and nucelotides. Indeed, the O-GlcNAc axis can also be regulated with a variety of other metabolites ranging from glucosamine to diets such as the ketogenic diet, which has been implemented in epilepsy cases [72-77]. The ketogenic diet has been demonstrated to aid in epilepsy through disruption of glutametergic signaling, inhibition of glycolysis and activation of ATP dependent potassium channels [78]. By favoring a high fat diet over carbohydrates such as in the ketogenic diet, the hexosamine biosynthesis pathway (HBP) production is promoted even with a sparsity of glucose [22, 79-81]. These studies have demonstrated that the upregulation of HBP, which is responsible for UDP-GlcNAc used by OGT, as well as the upregulation of OGT levels provides the combination of substrate availability and enzyme protein levels that lead to global increases in O-GlcNAcylation. 
An alternative method to increase O-GlcNAcylation used by Cheung et al, was the use of the FDA approved, economic, and widely used diabetic drug metformin. Metformin stimulates OGT increases via AMP-activated protein kinase (AMPK) signaling, and leads to increase neurofilament H O-GlcNAcylation, a protein that supports neuronal cytoskeletal structures. Our data provide interesting preliminary findings that merit further investigation between beneficial outcome of increased O-GlcNAc with epilepsy and modulating either through several drugs or metabolically through diet. Furthermore, O-GlcNAc signaling has been characterized in numerous pathologies outside of the nervous system [82-85].

To date, the study of protein O-GlcNAcylation has been limited to a few neurodegenerative disorders including, Alzheimer's disease (AD), and Parkinson's disease (PD) share overlapping proteomic characteristics with respect to O-GlcNAc and epilepsy.[15-18, 86, 87]. Altered O-GlcNAcylation levels in the brain was first described in AD studies, showing tauopathies, which is typically hyper-phosphorylated in AD, can be O-GlcNAcylated [14]. By promoting this modification in AD in vivo, it can effectively block tau hyperphosphorylation, decrease $\beta$-amyloid peptide levels and amyloid plaques as well as treat cognitive decline in AD [18]. Although epilepsy doesn't share the same proteomic hallmarks as $\mathrm{AD}, \mathrm{AD}$ can be associated with seizures in models of $\mathrm{AD}$ as well as in humans [88-91]. While speculative at this point, the phenotypic overlap between AD and epilepsy should be considered when attempting to discover novel proteomic mechanisms to characterize each disorder or novel targets that could be O-GlcNAcylated in each pathology.

The link between PD and protein O-GlcNAcylation was initially through THAP1, a DNA binding protein that regulates cell proliferation via gene transcription and mutations on this gene have lead to DYT6 dystonia [92]. THAP1 was found to associate with HCF-1 and OGT in order to promote transcription of cell proliferation factors. This prompted further research to link PD and OGlcNAc, which lead to studies investigating the role of protein OGlcNAcylation of a-synuclein, the hallmark protein of PD. Studies showed that indeed asynuclein can be O-GlcNAcylated and it results in an arrest of its aggregation [93]. In modifying a-synuclein with O-GlcNAcylation you also effectively block its degredation [94]. Furthermore, in postmortem individuals with PD, OGlcNAcylation is increased, and when this observation is mimiced in primary cortical neurons, the mechanism is associated with increased activation of mTOR [95]. The PD field provides insights as to how mTOR OGlcNAcylation behaves in a neurodegenerative disorder which could provide further insights as to how it could be behaving in epilepsy. In addition, mTOR phosphorylation has been previously assoicated with epilepsy, which highlights the need for further study into the role of O-GlcNAcylated mTOR in epilepsy [96-99].

Notably, our findings identified a novel molecular target, OGA, which can be successfully depressed by Thiamet-G in order to promote O-GlcNAcylation levels and decrease the number of seizures and spikes in vivo both in rats and in human tissue. Although chronic inhibition of OGA in epileptic rats did not prevent or reverse ventricular expansion, we observed that Thiamet-G treatment in epileptic animals and humans tissue could be used to reduce seizures and spike frequency. In future studies, it may be of interest to treat these rats with Thiamet-G during earlier stages of epilepsy pathogenesis, or during the onset of status 
epilepticus to investigate whether Thiamet-G has a better therapeutic effect in delaying or halting epileptogenesis.

In summary, our results suggest a new role for protein O-GlcNAcylation and OGT/OGA mediators in TLE. Homeostatic expression of O-GlcNAcylation appears to be necessary in order to reduce seizures. These findings shed new light on the disorder and warrant further mechanistic studies on novel molecular targets that we have discovered. Considering that protein OGlcNAcylation is interconnected with broader cellular metabolism, many exciting treatment programs could be employed such as diet to target O-GlcNAcylation's role in epileptic pathophysiology. These therapies would consequently be targeted at the cellular metabolism level in order to regain homeostatic levels of O-GlcNAcylation.

\section{Supplementary Material}

Refer to Web version on PubMed Central for supplementary material.

\section{Acknowledgments:}

We would like to thank Drs. Cristin Gavin and Jing Wang of the Evelyn F. McKnight Synaptic Plasticity Core at $\mathrm{UAB}$ for technical assistance with electrophysiology experiments. We would like also like to thank Dr. James Mobley for carrying out the mass spectrometry at University of Alabama at Birmingham (UAB) Mass Spectrometry/Proteomics Shared Facility. This work was supported by the Epilepsy Foundation, McNulty Civitan International Scientist Award, and the National Institute of Neurological Disorders and Stroke (NS090250).

\section{Abbreviations}

O-GlcNAc

TLE

PTM

OGT

OGA

LTD

KA

CA

DG

SiRNA

fEPSP

EPSP

HPLC

EEG
O-linked- $\beta-\mathrm{N}$-acetyl glucosamine

Temporal Lobe Epilepsy

post-translational modifications

O-GlcNAc Transferase

O-GlcNAcase

long-term depression

kainic acid

cornu ammonis

Dentate Gyrus

small interfering RNA

field excitatory postsynaptic potential

excitatory postsynaptic potential

High performance liquid chomrotagraphy

electroencephalograph 


$\begin{array}{ll}\text { SE } & \text { Status Epilepticus } \\ \text { MRI } & \text { Magnetic Resonance Imaging } \\ \text { SORL1 } & \text { Sortilin-Related Receptor } \\ \text { Tmod2 } & \text { tropomodulin 2 } \\ \text { LDL } & \text { low density lipid } \\ \text { RAP } & \text { receptor-associated protein } \\ \text { APP } & \text { amyloid precursor protein } \\ \text { AED } & \text { Anti-epileptic drugs } \\ \text { IP } & \text { intraperitoneal } \\ \text { ACSF } & \text { artificial cerebral spinal fluid } \\ \text { GO } & \text { Gene ontology } \\ \text { CSF } & \text { cerebral spinal fluid } \\ \text { PMI } & \text { Postmortem Interval } \\ \text { ABC } & \text { Alabama Brain Collection }\end{array}$

\section{References}

1. Liu XY, et al., Comparative proteomics and correlated signaling network of rat hippocampus in the pilocarpine model of temporal lobe epilepsy. Proteomics, 2008 8(3): p. 582-603. [PubMed: 18186018]

2. Meriaux C, et al., Human temporal lobe epilepsy analyses by tissue proteomics. Hippocampus, 2014 24(6): p. 628-42. [PubMed: 24449190]

3. Gass P, Kiessling M, and Bading H, Regionally selective stimulation of mitogen activated protein (MAP) kinase tyrosine phosphorylation after generalized seizures in the rat brain. Neurosci Lett, 1993 162(1-2): p. 39-42. [PubMed: 7510055]

4. Mielke K, et al., Activity and expression of JNK1, p38 and ERK kinases, c-Jun N-terminal phosphorylation, and c-jun promoter binding in the adult rat brain following kainate-induced seizures. Neuroscience, 1999 91(2): p. 471-83. [PubMed: 10366004]

5. Nateri AS, et al., ERK activation causes epilepsy by stimulating NMDA receptor activity. Embo j, 2007 26(23): p. 4891-901. [PubMed: 17972914]

6. Lubin FD, et al., Kainate mediates nuclear factor-kappa B activation in hippocampus via phosphatidylinositol-3 kinase and extracellular signal-regulated protein kinase. Neuroscience, 2005 133(4): p. 969-81. [PubMed: 15916859]

7. Stewart LT, et al., Acute Increases in Protein O-GlcNAcylation Dampen Epileptiform Activity in Hippocampus. J Neurosci, 2017 37(34): p. 8207-8215. [PubMed: 28760863]

8. Khidekel N, et al., Probing the dynamics of O-GlcNAc glycosylation in the brain using quantitative proteomics. Nat Chem Biol, 2007 3(6): p. 339-48. [PubMed: 17496889]

9. Zachara NE and Hart GW, Cell signaling, the essential role of O-GlcNAc! Biochimica et Biophysica Acta (BBA) - Molecular and Cell Biology of Lipids, 2006 1761(5-6): p. 599-617. [PubMed: 16781888]

10. Hart GW, et al., Cross Talk Between O-GlcNAcylation and Phosphorylation: Roles in Signaling, Transcription, and Chronic Disease. Annual Review of Biochemistry, 2011 80: p. 825-858. 
11. Bond MR and Hanover JA, A little sugar goes a long way: The cell biology of O-GlcNAc. The Journal of Cell Biology, 2015 208(7): p. 869-880. [PubMed: 25825515]

12. Zachara NE, et al., Dynamic O-GlcNAc Modification of Nucleocytoplasmic Proteins in Response to Stress A SURVIVAL RESPONSE OF MAMMALIAN CELLS. Journal of Biological Chemistry, 2004 279(29): p. 30133-30142. [PubMed: 15138254]

13. Copeland RJ, Han G, and Hart GW, O-GlcNAcomics--Revealing roles of OGlcNAcylation in disease mechanisms and development of potential diagnostics. Proteomics Clin Appl, 2013 7(910): p. 597-606. [PubMed: 23640805]

14. Yuzwa SA, et al., A potent mechanism-inspired O-GlcNAcase inhibitor that blocks phosphorylation of tau in vivo. Nat Chem Biol, 2008 4(8): p. 483-90. [PubMed: 18587388]

15. Gatta E, et al., Evidence for an imbalance between tau O-GlcNAcylation and phosphorylation in the hippocampus of a mouse model of Alzheimer's disease. Pharmacological research, 2016 105: p. 186-197. [PubMed: 26816085]

16. Wani WY, et al., O-GlcNAcylation and neurodegeneration. Brain Res Bull, 2017 133: p. 80-87. [PubMed: 27497832]

17. Xie S, et al., O-GlcNAcylation of protein kinase A catalytic subunits enhances its activity: a mechanism linked to learning and memory deficits in Alzheimer's disease. Aging cell, 2016.

18. Yuzwa SA, et al., Pharmacological inhibition of O-GlcNAcase (OGA) prevents cognitive decline and amyloid plaque formation in bigenic tau/APP mutant mice. Molecular Neurodegeneration, 2014 9: p. 42. [PubMed: 25344697]

19. Cole RN and Hart GW, Cytosolic O-glycosylation is abundant in nerve terminals. Journal of Neurochemistry, 2001 79(5): p. 1080-1089. [PubMed: 11739622]

20. Lagerlof $\mathrm{O}$, et al., The nutrient sensor OGT in PVN neurons regulates feeding. Science, 2016 351(6279): p. 1293-6. [PubMed: 26989246]

21. Taylor EW, et al., O-GlcNAcylation of AMPA receptor GluA2 is associated with a novel form of long-term depression at hippocampal synapses. J Neurosci, 2014 34(1): p. 10-21. [PubMed: 24381264]

22. Cheung WD and Hart GW, AMP-activated Protein Kinase and p38 MAPK Activate OGlcNAcylation of Neuronal Proteins during Glucose Deprivation. Journal of Biological Chemistry, 2008 283(19): p. 13009-13020. [PubMed: 18353774]

23. Pekkurnaz G, et al., Glucose regulates mitochondrial motility via Milton modification by OGlcNAc transferase. Cell, 2014 158(1): p. 54-68. [PubMed: 24995978]

24. Lagerlof O, Hart GW, and Huganir RL, O-GlcNAc transferase regulates excitatory synapse maturity. Proc Natl Acad Sci U S A, 2017 114(7): p. 1684-1689. [PubMed: 28143929]

25. Racine RJ, Modification of seizure activity by electrical stimulation. I. After-discharge threshold. Electroencephalogr Clin Neurophysiol, 1972 32(3): p. 269-79. [PubMed: 4110396]

26. Ferrer I, et al., Brain Protein Preservation Largely Depends on the Postmortem Storage Temperature: Implications for Study of Proteins in Human Neurologic Diseases and Management of Brain Banks: A BrainNet Europe Study. Journal of Neuropathology \& Experimental Neurology, 2007 66(1): p. 35-46. [PubMed: 17204935]

27. McCullumsmith RE, et al., Postmortem Brain: An Underutilized Substrate for Studying Severe Mental Illness. Neuropsychopharmacology, 2014 39(1): p. 65-87. [PubMed: 24091486]

28. Weatherly DB, et al., A Heuristic method for assigning a false-discovery rate for protein identifications from Mascot database search results. Mol Cell Proteomics, 2005 4(6): p. 762-72. [PubMed: 15703444]

29. Keller A, et al., Empirical statistical model to estimate the accuracy of peptide identifications made by MS/MS and database search. Anal Chem, 2002 74(20): p. 5383-92. [PubMed: 12403597]

30. Hensley K, et al., Membrane protein alterations in rodent erythrocytes and synaptosomes due to aging and hyperoxia. Biochim Biophys Acta, 1995 1270(2-3): p. 203-6. [PubMed: 7727544]

31. Liu H, Sadygov RG, and Yates JR, 3rd, A model for random sampling and estimation of relative protein abundance in shotgun proteomics. Anal Chem, 2004 76(14): p. 4193-201. [PubMed: 15253663]

32. Old WM, et al., Comparison of label-free methods for quantifying human proteins by shotgun proteomics. Mol Cell Proteomics, 2005 4(10): p. 1487-502. [PubMed: 15979981] 
33. Beissbarth T, et al., Statistical modeling of sequencing errors in SAGE libraries. Bioinformatics, 200420 Suppl 1: p. i31-9. [PubMed: 15262778]

34. Golub TR, et al., Molecular classification of cancer: class discovery and class prediction by gene expression monitoring. Science, 1999 286(5439): p. 531-7. [PubMed: 10521349]

35. Xu W, et al., Serum profiling by mass spectrometry combined with bioinformatics for the biomarkers discovery in diffuse large B-cell lymphoma. Tumour Biol, 2015 36(3): p. 2193-9. [PubMed: 25409615]

36. Bhatia VN, et al., Software tool for researching annotations of proteins: open-source protein annotation software with data visualization. Anal Chem, 2009 81(23): p. 9819-23. [PubMed: 19839595]

37. Ekins S, et al., Algorithms for network analysis in systems-ADME/Tox using the MetaCore and MetaDrug platforms. Xenobiotica, 2006 36(10-11): p. 877-901. [PubMed: 17118913]

38. Roopun AK, et al., A nonsynaptic mechanism underlying interictal discharges in human epileptic neocortex. Proc Natl Acad Sci U S A, 2010 107(1): p. 338-43. [PubMed: 19966298]

39. Cunningham MO, et al., Glissandi: transient fast electrocorticographic oscillations of steadily increasing frequency, explained by temporally increasing gap junction conductance. Epilepsia, 2012 53(7): p. 1205-14. [PubMed: 22686654]

40. Simon A, et al., Gap junction networks can generate both ripple-like and fast ripple-like oscillations. Eur J Neurosci, 2014 39(1): p. 46-60. [PubMed: 24118191]

41. Webb WM, et al., Dynamic association of epigenetic H3K4me3 and DNA 5hmC marks in the dorsal hippocampus and anterior cingulate cortex following reactivation of a fear memory. Neurobiol Learn Mem, 2017 142(Pt A): p. 66-78. [PubMed: 28232238]

42. Cahn BR and Polich J, Meditation states and traits: EEG, ERP, and neuroimaging studies. Psychol Bull, 2006 132(2): p. 180-211. [PubMed: 16536641]

43. Fisher RS, et al., High-frequency EEG activity at the start of seizures. J Clin Neurophysiol, 1992 9(3): p. 441-8. [PubMed: 1517412]

44. Worrell GA, et al., High-frequency oscillations and seizure generation in neocortical epilepsy. Brain, 2004 127(7): p. 1496-1506. [PubMed: 15155522]

45. Haddad T, et al., Temporal epilepsy seizures monitoring and prediction using cross-correlation and chaos theory. Healthcare Technology Letters, 2014 1(1): p. 45-50. [PubMed: 26609376]

46. Al Sufiani F and Ang LC, Neuropathology of Temporal Lobe Epilepsy. Epilepsy Research and Treatment, 2012 2012: p. 13.

47. Crespel A, et al., Inflammatory reactions in human medial temporal lobe epilepsy with hippocampal sclerosis. Brain Res, 2002 952(2): p. 159-69. [PubMed: 12376176]

48. Engel J, Jr., Surgery for seizures. N Engl J Med, 1996 334(10): p. 647-52. [PubMed: 8592530]

49. Sofroniew MV and Vinters HV, Astrocytes: biology and pathology. Acta Neuropathologica, 2010 119(1): p. 7-35. [PubMed: 20012068]

50. Thom D, Dilatiation of the lateral ventricles as a common brain lesion in epilepsy. H. Nerv Ment Dis, 1917 46: p. 355-358.

51. Fuerst D, et al., Hippocampal sclerosis is a progressive disorder: a longitudinal volumetric MRI study. Ann Neurol, 2003 53(3): p. 413-6. [PubMed: 12601713]

52. Dabbs K, et al., Brain structure and aging in chronic temporal lobe epilepsy. Epilepsia, 2012 53(6): p. 1033-43. [PubMed: 22471353]

53. Coulter DA and Steinhauser C, Role of astrocytes in epilepsy. Cold Spring Harb Perspect Med, 2015 5(3): p. a022434. [PubMed: 25732035]

54. Wieshmann UC, et al., Development of hippocampal atrophy: a serial magnetic resonance imaging study in a patient who developed epilepsy after generalized status epilepticus. Epilepsia, 1997 38(11): p. 1238-41. [PubMed: 9579926]

55. Jackson DC, et al., Ventricular enlargement in new-onset pediatric epilepsies. Epilepsia, 2011 52(12): p. 2225-32. [PubMed: 22091816]

56 . $\mathrm{Bu} \mathrm{G}$, The roles of receptor-associated protein (RAP) as a molecular chaperone for members of the LDL receptor family. Int Rev Cytol, 2001 209: p. 79-116. [PubMed: 11580203] 
57. Zollo A, et al., Sortilin-Related Receptor Expression in Human Neural Stem Cells Derived from Alzheimer's Disease Patients Carrying the APOE Epsilon 4 Allele. Neural Plast, 2017 2017: p. 1892612. [PubMed: 28634550]

58. Yin RH, Yu JT, and Tan L, The Role of SORL1 in Alzheimer's Disease. Mol Neurobiol, 2015 51(3): p. 909-18. [PubMed: 24833601]

59. Yang JW, et al., Aberrant expression of cytoskeleton proteins in hippocampus from patients with mesial temporal lobe epilepsy. Amino Acids, 2006 30(4): p. 477-93. [PubMed: 16583313]

60. Sussman MA, et al., Neural tropomodulin: developmental expression and effect of seizure activity. Brain Res Dev Brain Res, 1994 80(1-2): p. 45-53. [PubMed: 7955359]

61. Tallent MK, et al., In vivo modulation of O-GlcNAc levels regulates hippocampal synaptic plasticity through interplay with phosphorylation. J Biol Chem, 2009 284(1): p. 174-81. [PubMed: 19004831]

62. Kanno T, et al., Regulation of AMPA receptor trafficking by O-glycosylation. Neurochem Res, 2010 35(5): p. 782-8. [PubMed: 20165912]

63. Wu W. g., et al., Structural Study on O-Glycopeptides: Glycosylation-Induced Conformational Changes of O-GlcNAc, O-LacNAc, O-Sialyl-LacNAc, and O-Sialyl-Lewis-X Peptides of the Mucin Domain of MAdCAM-1. Journal of the American Chemical Society, 1999 121(11): p. 2409-2417.

64. Liang FC, et al., Tuning the conformation properties of a peptide by glycosylation and phosphorylation. Biochem Biophys Res Commun, 2006 342(2): p. 482-8. [PubMed: 16487934]

65. Butkinaree C, Park K, and Hart GW, O-linked beta-N-acetylglucosamine (O-GlcNAc): Extensive crosstalk with phosphorylation to regulate signaling and transcription in response to nutrients and stress. Biochim Biophys Acta, 2010 1800(2): p. 96-106. [PubMed: 19647786]

66. Wang Z, Gucek M, and Hart GW, Cross-talk between GlcNAcylation and phosphorylation: sitespecific phosphorylation dynamics in response to globally elevated OGlcNAc. Proc Natl Acad Sci U S A, 2008 105(37): p. 13793-8. [PubMed: 18779572]

67. Wang Z, Pandey A, and Hart GW, Dynamic interplay between O-linked N-acetylglucosaminylation and glycogen synthase kinase-3-dependent phosphorylation. Mol Cell Proteomics, 2007 6(8): p. 1365-79. [PubMed: 17507370]

68. Xu J, et al., Regulation of the Proteasome by AMPK in Endothelial Cells: The Role of OGlcNAc Transferase (OGT). PLoS ONE, 2012 7(5): p. e36717. [PubMed: 22574218]

69. Ozcan S, Andrali SS, and Cantrell JE, Modulation of transcription factor function by OGlcNAc modification. Biochim Biophys Acta, 2010 1799(5-6): p. 353-64. [PubMed: 20202486]

70. Sun Y, et al., A gel-based proteomic method reveals several protein pathway abnormalities in fetal Down syndrome brain. J Proteomics, 2011 74(4): p. 547-57. [PubMed: 21262400]

71. Chen A, et al., Upregulation of dihydropyrimidinase-related protein 2, spectrin alpha II chain, heat shock cognate protein 70 pseudogene 1 and tropomodulin 2 after focal cerebral ischemia in rats--a proteomics approach. Neurochem Int, 2007 50(7-8): p. 1078-86. [PubMed: 17196711]

72. Walgren JL, et al., High glucose and insulin promote O-GlcNAc modification of proteins, including alpha-tubulin. Am J Physiol Endocrinol Metab, 2003 284(2): p. E424-34. [PubMed: 12397027]

73. Housley MP, et al., O-GlcNAc regulates FoxO activation in response to glucose. J Biol Chem, 2008 283(24): p. 16283-92. [PubMed: 18420577]

74. Kang ES, et al., O-GlcNAc modulation at Akt1 Ser473 correlates with apoptosis of murine pancreatic beta cells. Exp Cell Res, 2008 314(11-12): p. 2238-48. [PubMed: 18570920]

75. Lima VV, et al., High fat diet increases O-GlcNAc levels in cerebral arteries: a link to vascular dysfunction associated with hyperlipidemia/obesity? Clinical science (London, England : 1979), 2016 130(11): p. 871-880.

76. Rogovik AL and Goldman RD, Ketogenic diet for treatment of epilepsy. Canadian Family Physician, 2010 56(6): p. 540-542. [PubMed: 20547519]

77. de Lima PA, de Brito Sampaio LP, and Damasceno NRT, Neurobiochemical mechanisms of a ketogenic diet in refractory epilepsy. Clinics, 2014 69(10): p. 699-705. [PubMed: 25518023]

78. Lutas A and Yellen G, The ketogenic diet: metabolic influences on brain excitability and epilepsy. Trends in neurosciences, 2013 36(1): p. 32-40. [PubMed: 23228828] 
79. Zou L, et al., Glucose deprivation-induced increase in protein O-GlcNAcylation in cardiomyocytes is calcium-dependent. J Biol Chem, 2012 287(41): p. 34419-31. [PubMed: 22908225]

80. Taylor RP, et al., Glucose deprivation stimulates O-GlcNAc modification of proteins through upregulation of O-linked N-acetylglucosaminyltransferase. J Biol Chem, 2008 283(10): p. 6050-7. [PubMed: 18174169]

81. Chaveroux C, et al., Nutrient shortage triggers the hexosamine biosynthetic pathway via the GCN2-ATF4 signalling pathway. Sci Rep, 2016 6: p. 27278. [PubMed: 27255611]

82. Macauley MS, et al., Elevation of Global O-GlcNAc in Rodents Using a Selective OGlcNAcase Inhibitor Does Not Cause Insulin Resistance or Perturb Glucohomeostasis. Chemistry \& Biology, 2010 17(9): p. 949-958. [PubMed: 20851344]

83. Champattanachai V, Marchase RB, and Chatham JC, Glucosamine protects neonatal cardiomyocytes from ischemia-reperfusion injury via increased protein-associated OGlcNAc. Am J Physiol Cell Physiol, 2007 292(1): p. C178-87. [PubMed: 16899550]

84. Singh JP, et al., O-GlcNAc signaling in cancer metabolism and epigenetics. Cancer Lett, 2015 356(2 Pt A): p. 244-50. [PubMed: 24769077]

85. Lewis BA and Hanover JA, O-GlcNAc and the Epigenetic Regulation of Gene Expression.

86. Narayan S, et al., Evidence for disruption of sphingolipid metabolism in schizophrenia. J Neurosci Res, 2009 87(1): p. 278-88. [PubMed: 18683247]

87. Hung WY, Mold DE, and Tourian A, Huntington's-chorea fibroblasts. Cellular protein glycosylation. Biochem J, 1980 190(3): p. 711-9. [PubMed: 6451218]

88. Liu D, et al., Brain regional synchronous activity predicts tauopathy in 3xTgAD mice. Neurobiol Aging, 2018 70: p. 160-169. [PubMed: 30015035]

89. Hall AM, et al., Tau-dependent Kv4.2 depletion and dendritic hyperexcitability in a mouse model of Alzheimer's disease. J Neurosci, 2015 35(15): p. 6221-30. [PubMed: 25878292]

90. Haberman RP, Koh MT, and Gallagher M, Heightened cortical excitability in aged rodents with memory impairment. Neurobiology of Aging, 2017 54: p. 144-151. [PubMed: 28104309]

91. Born HA, Seizures in Alzheimer's disease. Neuroscience, 2015 286: p. 251-263. [PubMed: 25484360]

92. Mazars R, et al., The THAP-Zinc Finger Protein THAP1 Associates with Coactivator HCF-1 and O-GlcNAc Transferase: A LINK BETWEEN DYT6 AND DYT3 DYSTONIAS. The Journal of Biological Chemistry, 2010 285(18): p. 13364-13371. [PubMed: 20200153]

93. Marotta NP, et al., O-GlcNAc modification blocks the aggregation and toxicity of the protein alpha-synuclein associated with Parkinson's disease. Nat Chem, 2015 7(11): p. 913-20. [PubMed: 26492012]

94. Levine PM, et al., O-GlcNAc modification inhibits the calpain-mediated cleavage of a-synuclein. Bioorganic \& Medicinal Chemistry, 2017 25(18): p. 4977-4982.

95. Wani WY, et al., O-GlcNAc regulation of autophagy and alpha-synuclein homeostasis; implications for Parkinson's disease. Mol Brain, 2017 10(1): p. 32. [PubMed: 28724388]

96. Cork GK, Thompson J, and Slawson C, Real Talk: The Inter-play Between the mTOR, AMPK, and Hexosamine Biosynthetic Pathways in Cell Signaling. Front Endocrinol (Lausanne), 2018 9: p. 522. [PubMed: 30237786]

97. Citraro R, et al., mTOR pathway inhibition as a new therapeutic strategy in epilepsy and epileptogenesis. Pharmacol Res, 2016 107: p. 333-343. [PubMed: 27049136]

98. Crino PB, The mTOR signalling cascade: paving new roads to cure neurological disease. Nat Rev Neurol, 2016 12(7): p. 379-92. [PubMed: 27340022]

99. Duan W, Chen Y, and Wang XR, MicroRNA155 contributes to the occurrence of epilepsy through the PI3K/Akt/mTOR signaling pathway. Int J Mol Med, 2018 42(3): p. 1577-1584. [PubMed: 29901097] 


\section{Highlights}

- O-GlcNAcylation and OGT are decreased in the epileptic hippocampus of rodents and TLE patients.

- $\quad$ Promoting O-GlcNAcylation decreases epileptiform activity.

- $\quad$ Novel proteins associated with O-GlcNAcylation in the epileptic hippocampus.

- Targeting O-GlcNAcylation should be considered as a novel treatment for seizure control. 
b

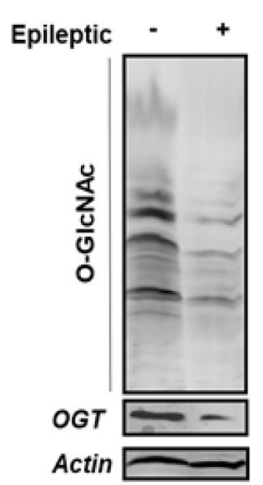

a

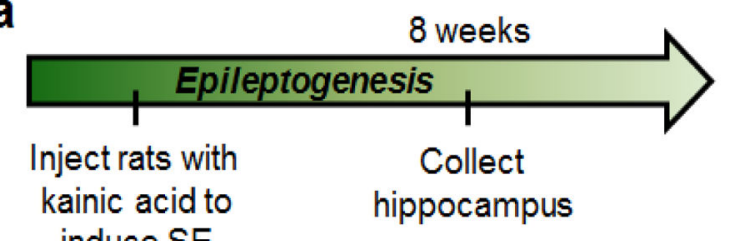

induce SE

C

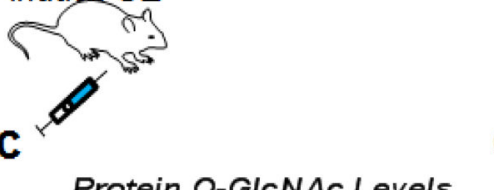

in hippocampal CA regions

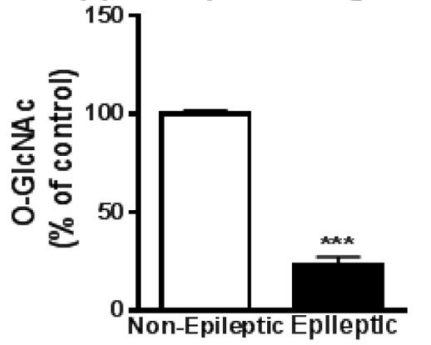

d

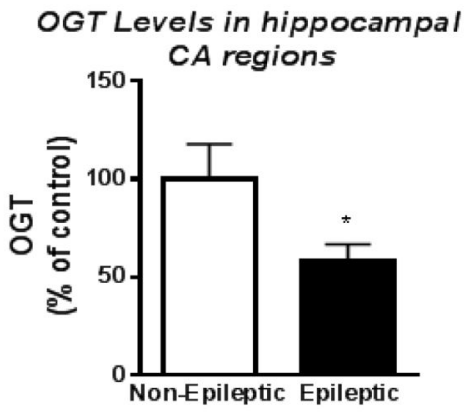

Figure 1: O-GIcNAcylation and OGT levels decreased in the hippocampus of epileptic rats. (a) Experimental design. Rats were either injected with saline or kainic acid in order to induce status epilepticus (SE). The animals were then sacrificed eight weeks post-KA injection at which point these animals had become epileptic and the hippocampus was collected for protein analysis. (b) Representative O-GlcNAcylation as well as OGT and actin western blots for controls and epileptic rats. (c) Global O-GlcNAcylation was decreased in epileptic rats in comparison to control. ( $n=4-6$ per group) (d) OGT protein levels were significantly reduced in epilepsy ( $\mathrm{n}=4-6$ per group). .* denotes $\mathrm{P}<0.05$ from controls, *** denotes $\mathrm{P}<0.001$ from controls. Unpaired T-Test Error bars are SEM 
a

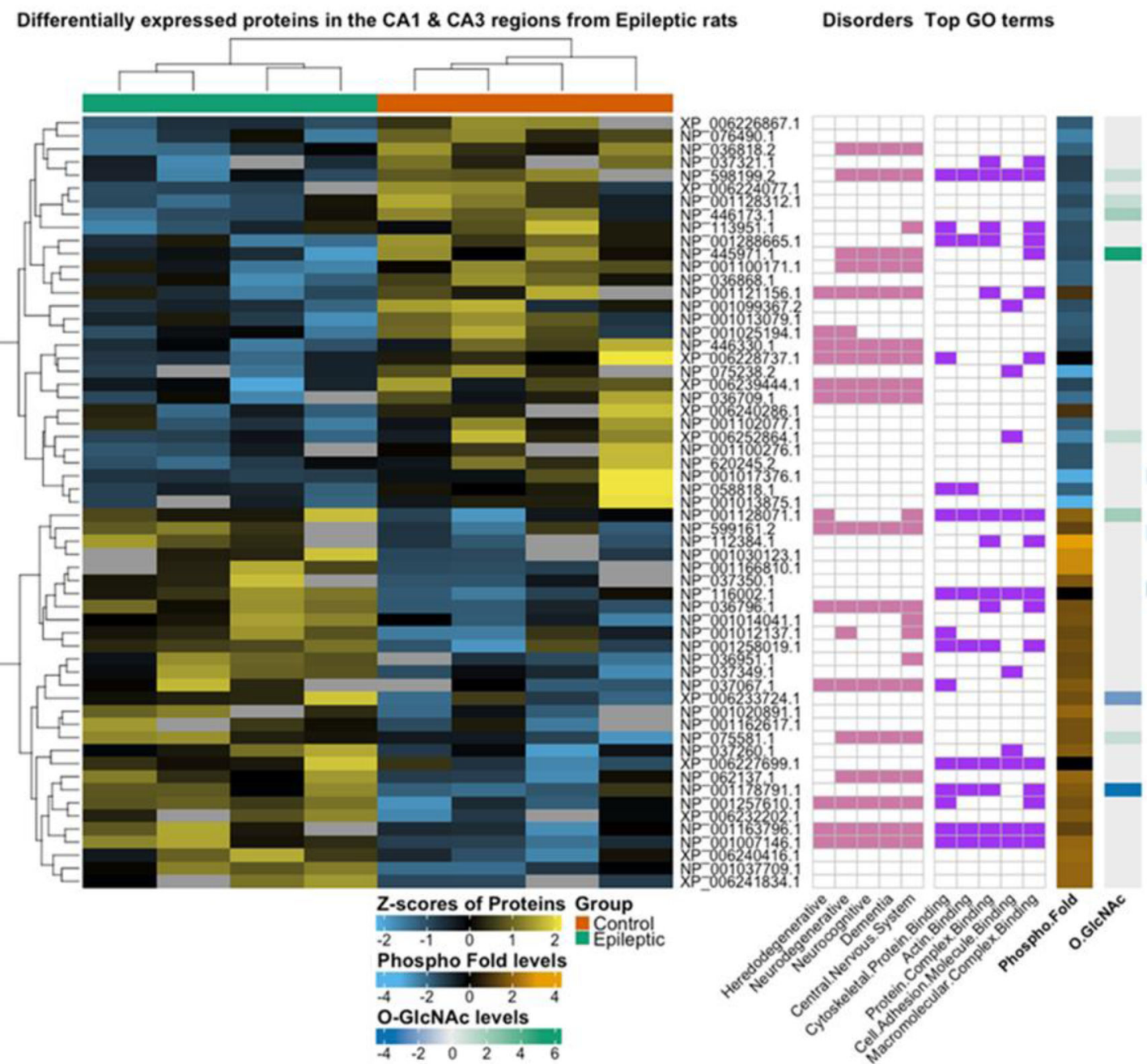

Figure 2: Protein expression and PTM in epilepsy.

(a) The heatmap illustrates all differentially expressed proteins $(\mathrm{p}<0.05)$ in epileptic rats (green bar) relative to controls (orange) bar. Each row is a protein indicated by the RefSeq accession number and each column in a biological replicate where the row and column order was determined by the Euclidian clustering method shown by the dendrograms. The protein values are shown as standardized $\mathrm{z}$-scores, where the color indicates the standard deviation increasing (yellow) or decreasing (blue) relative to the mean (black). Grey blocks indicate missing values for the respective biological replicate. Further, for each protein, the top five disorders and GO terms (adjusted p-value $<0.05$ ) are annotated in pink and purple respectively. Lastly, the phosphorylation (phospho) fold change and O-GlcNAc levels are indicated for each differentially expressed protein.* denotes $\mathrm{P}<0.05$ from controls, *** denotes $\mathrm{P}<0.001$ from controls. Unpaired T-Test Error bars are SEM 


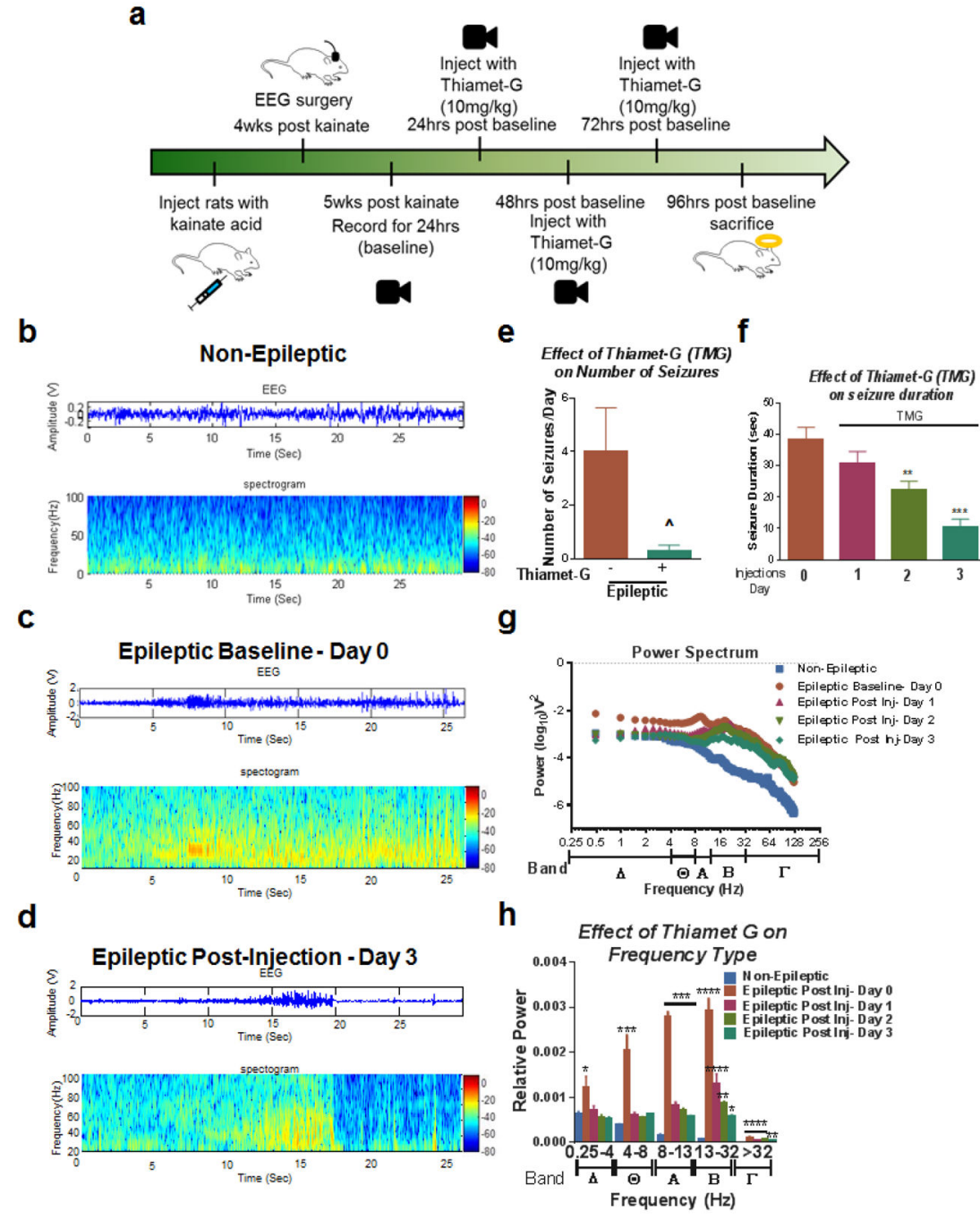

Figure 3: OGA inhibition decreased seizure duration and epileptiform activity.

(a) Experimental outline. Epileptic rats were created using kainic acid. Four weeks post kainate the rats underwent EEG surgery where cortical electrodes were placed and the animals had a week to recover from the surgery before recordings were initiated. Baseline recordings were taking for $24 \mathrm{hrs}$ and Thiamet $-\mathrm{G}$ treatment ensued immediately after for three consecutive days followed by euthanization. (b) Cortical baseline EEG spectrogram of a saline (control) treated rat. (c) Cortical baseline EEG spectrogram of an epileptic rat during a seizure. (d) Cortical EEG spectrogram of the same epileptic rat following three days of Thiamet-G treatment. (e) The number of seizures decreased after three days of Thiamet-G treatment between the pre and post-treated animals. (f) Thiamet-G significantly decreased seizure duration by the second day of treatment and continued to decrease seizure duration up to the last day of treatment. (g) A power spectrum analysis demonstrated that the frequencies that were most dampened by Thiamet-G intervention were theta through gamma bands (h) Quantification of the power spectrum illustrates which frequencies were significantly decreased after treatment in comparison to control non-epileptic animals. * denotes $\mathrm{P}<0.05$ from controls, ** denotes $\mathrm{P}<0.01$ from controls, *** denotes $\mathrm{P}<0.001$ from 
controls, $* * * *$ denotes $\mathrm{P}<0.0001$ from controls. ${ }^{\wedge}$ denotes $\mathrm{P}<0.10$ One-way ANOVA, Error bars are SEM 


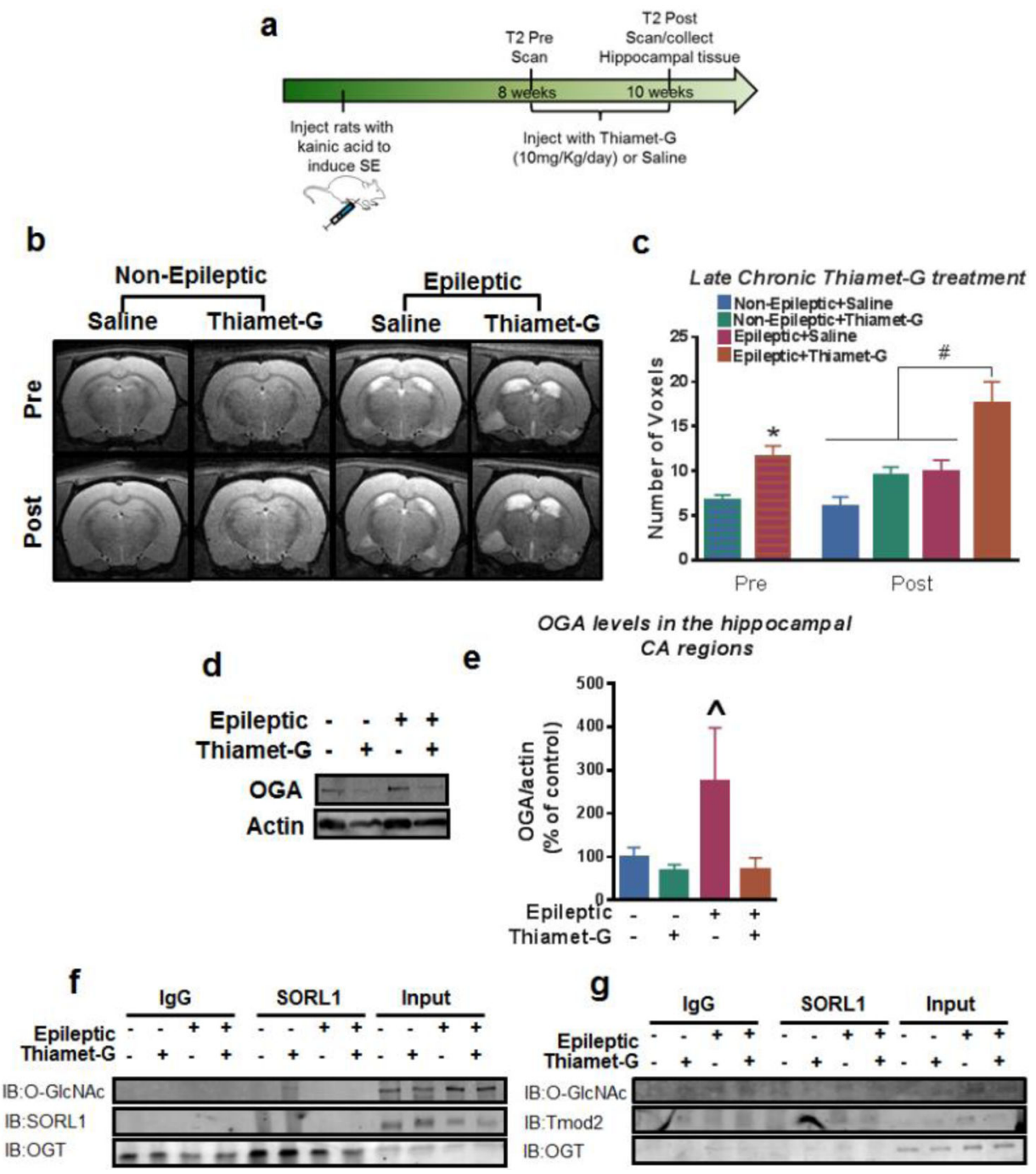

Figure 4: Thiamet-G treatment has no reduction in ventricle expansion or protein specific changes in O-GlcNAcylation.

(a) Experimental outline of animal model and treatment. Epileptic animals were created with kainic acid. Eight weeks post-kainate the animals had their first T2 scans were taken. Immediately following the scan, animals were treated with Thiamet-G (10mg/kg/day) for 2 weeks at the same time each day. The animals then had a final T2 scan where they were then sacrificed and the hippocampus was collected. (b)Representative pre/post T2 weighted images of epileptic and non-epileptic rats that were treated with either saline or Thiamet-G for two weeks. The CSF is bright white in the T2 MRI images demonstrating ventricle expansion with epilepsy and a more severe expansion with Thiamet-G treatment. (c) Quantification of T2 MRI images showing significant ventricle sizes between controls and epileptics before Thiamet-G treatment. Ventricle sizes significantly differed between the epileptic Thiamet-G treated group and the rest of the other group's post-treatment. $(n=8 /$ group). (d) Representative western blots of OGA and actin for the two-week saline or Thiamet-G treated epileptic and non-epileptic rats. (e) statistical analysis of the two-week saline or Thiamet-G treated epileptic and non-epileptic rats normalized to actin. (f) 
Immunoprecipitation of SORL1 with immunoblotting for O-GlcNAc (top membrane), SORL1 (middle membrane), and OGT (bottom membrane). (g) Immunoprecipitation of Tmod2 with immunoblotting for O-GlcNAc (top membrane), Tmod2 (middle membrane), and OGT bottom (membrane). (n=6-7/group) * denotes $\mathrm{P}<0.05$ from Non-epileptic plus saline controls. ${ }^{\#}$ denotes $\mathrm{P}<0.05$ from Epileptic plus Thiamet-G. ${ }^{\wedge}$ denotes $\mathrm{P}<0.10$ from Nonepileptic plus saline controls. One-way ANOVA. Error bars are SEM. 
a

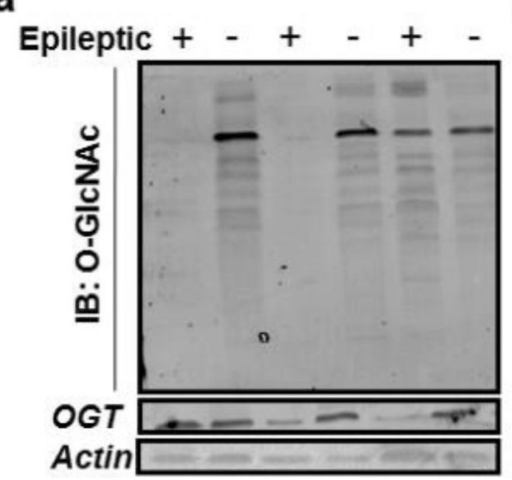

b

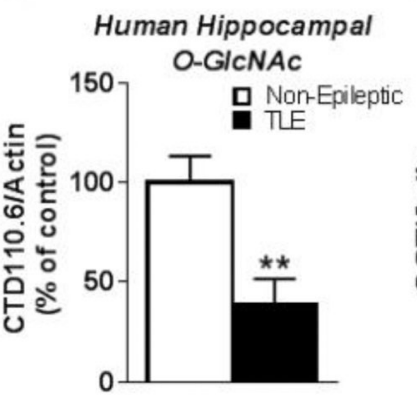

C

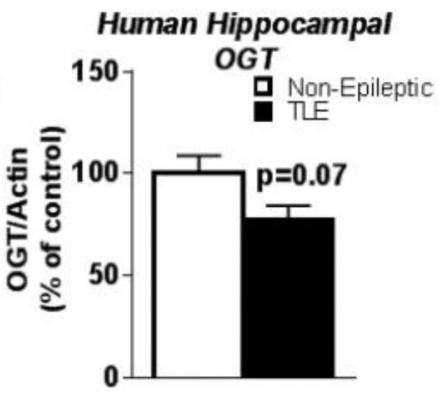

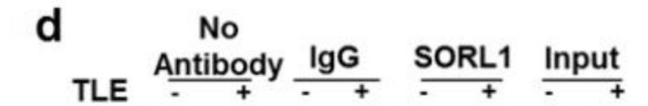

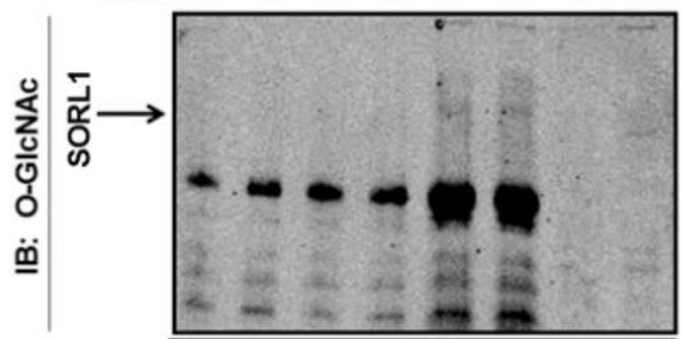

IB: SORL1

IB: OGT

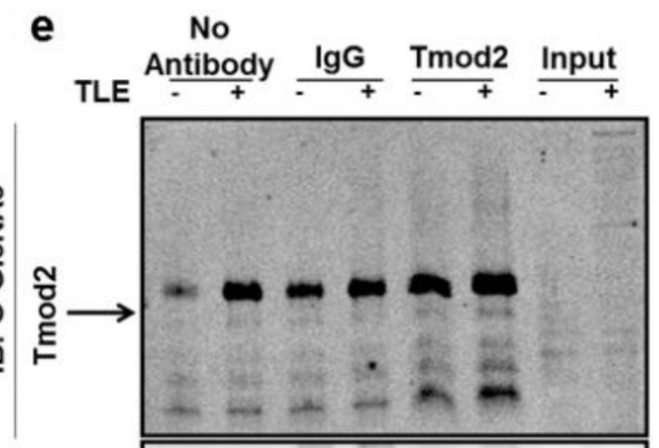

IB: Tmod2

IB: OGT

Figure 5: Tissues from patients with TLE have significant deficits in O-GlcNAcylation and OGT (a) Western blot membrane with TLE human tissue and post-mortem non-epileptic alternating from left to right. The top membrane was probed with CTD110.6 antibody to show O-GlcNAc levels between both groups. The middle membrane was stripped and probed with OGT and the bottom membrane represents the level of actin between both groups. (b) Desensitization of O-GlcNAc levels between control and TLE individuals were quantified and actin was used to normalize O-GlcNAc. ( $\mathrm{n}=11-13$ per group) (c) Desensitization of OGT levels between control and TLE where taken and normalized to actin. ( $\mathrm{n}=11-13$ per group). Immunoprecipitation of (d) SORL1 and (e) Tmod2 on resected TLE patients and postmortem tissue. Immunoblotting was performed with O-GlcNAc (top membrane), SORL1 (middle membrane), Tmod2 (middle membrane), and OGT (bottom membrane). Unpaired T-Test. ${ }^{* *}$ denotes $P<0.01$. Error bars are SEM. 
a

Bath applied
Shicing and acclimation to

Oxygenated ACSF During recording
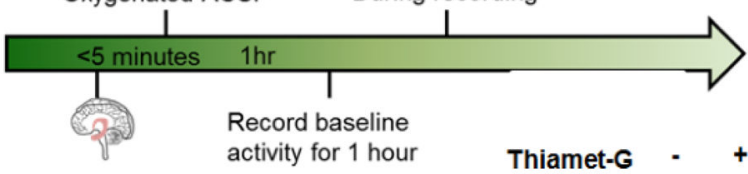

activity for 1 hour

Focal Site

Resection

b

Pre Thiamet-G treatment

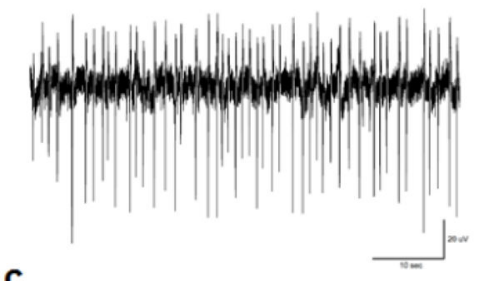

C

Post Thiamet-G treatment

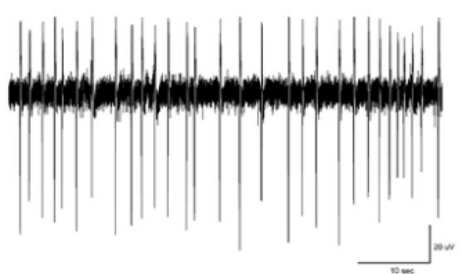

f

e
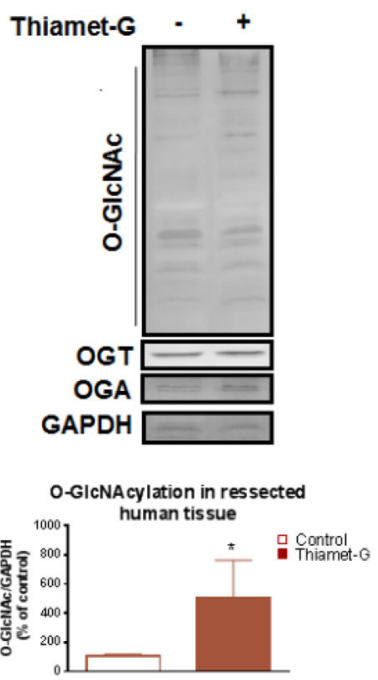

d
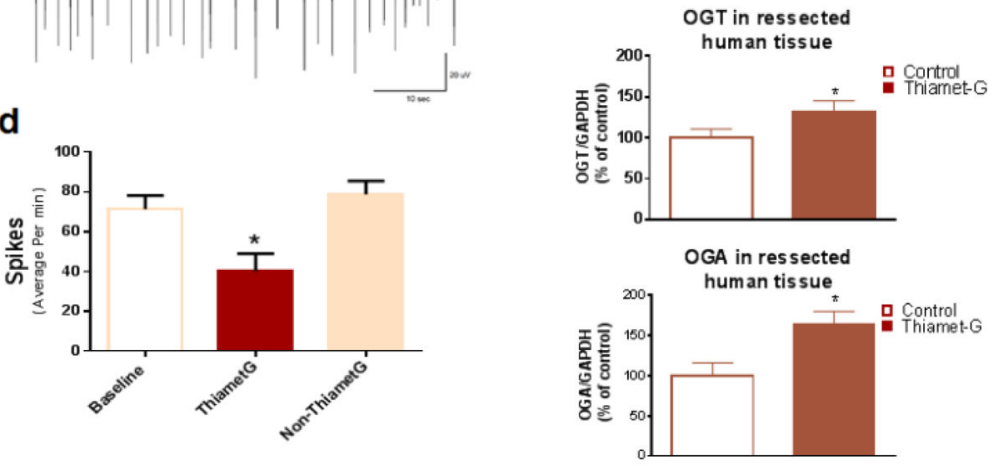

Figure 6: Thiamet-G bath application on human resectedtissue reduced interictal-like activity and increased OGT, and OGA protein expression.

(a) Experimental outlined. Samples were taken from patients that had undergone temporal lobectomy. Samples were immediately placed in oxygenated ACSF and allowed to acclimate for $1 \mathrm{hr}$. Baseline recording of activity was taken for $1 \mathrm{hr}$ followed by bath application of Thiamet-G. Samples were flash frozen and stored at $-80^{\circ} \mathrm{C}$. (b) Representative extracellular recording following Thiamet-G administration (c) Representative extracellular recording following Thiamet-G bath application. (d) Quantification of spiking events from tissue slices at baseline and following Thiamet-G administration. (e) Protein O-GlcNAcylation OGT and OGA representative western blots. (f) Quantification of western blots in Panel e where OGlcNAc, OGT, and OGA were normalized to actin and compared to untreated controls $(n=4 /$ group). * denotes $\mathrm{P}<0.05$. Fisher LSD test. Error bars are SEM. 